\title{
Alteration of renal functional, oxidative stress and inflammatory indices following hepatic ischemia-reperfusion
}

\author{
Mehri Kadkhodaee ${ }^{1}$, Saideh Mikaeili ${ }^{1}$, Maryam Zahmatkesh ${ }^{2}$, Freshteh Golab ${ }^{1}$, Behjat Seifi, \\ Hossein-Ali Arab ${ }^{3}$, Sedigheh Shams ${ }^{4}$ and Mitra Mahdavi-Mazdeh ${ }^{5}$ \\ ${ }^{1}$ Department of Physiology, School of Medicine, Tehran University of Medical Sciences, Tehran, Iran \\ ${ }^{2}$ Department of Neuroscience, School of Advanced Medical Technologies, Tehran University of Medical Sciences, Tehran, Iran \\ ${ }^{3}$ Department of Pharmacology, Faculty of Veterinary, Tehran University, Tehran, Iran \\ ${ }^{4}$ Children Medical Center, School of Medicine, Tehran University of Medical Sciences, Tehran, Iran \\ ${ }^{5}$ Department of Nephrology, School of Medicine, Tehran University of Medical Sciences, Tehran, Iran
}

\begin{abstract}
Liver ischemia/reperfusion (IR) injury is a complex phenomenon that may cause local as well as remote organ injuries. Reactive oxygen species (ROS) along with many pro- and anti- inflammatory cytokines are implicated in the development of organ injury. The renal functional, histological, oxidative stress and inflammatory indices were studied during a short and a longer period of liver IR. Rats were subjected to either sham operation or $90 \mathrm{~min}$ partial liver ischemia followed by 4 or $24 \mathrm{~h}$ of reperfusion. Serum ALT, AST, ALK and LDH levels, BUN and creatinine, renal MDA level, SOD and catalase activities were evaluated as well as serum IL- 6 and IL-10 concentrations along with renal histological evaluation. Ninety minutes liver ischemia $/ 4 \mathrm{~h}$ reperfusion caused an increase in BUN and renal MDA levels and a decrease in SOD and catalase activities. It also caused an increase in serum IL-6 and IL-10 levels. $24 \mathrm{~h}$ liver reperfusion resulted in a reduction in BUN levels and lower oxidative damages demonstrated by a decrease in renal MDA levels and an increase in renal SOD and catalase activities comparing to $4 \mathrm{~h}$ reperfusion group. Evaluations indicated improvement in histology such as less cytoplasmic vacuolation and lower tubular debris. Serum inflammatory indices (IL-6 and IL-10 levels) were also reduced. This study showed that liver IR damage causes renal injury including functional, inflammatory and oxidative status changes. The remote kidney damage was then improved by continuing reperfusion from 4 to $24 \mathrm{~h}$.
\end{abstract}

Key words: Liver - Ischemia/reperfusion - Remote organ - Kidney - ROS - Oxidative stress

\begin{abstract}
Abbreviations: ALK, alkaline phosphatase; ALT, alanine amino-transferase; AST, aspartate aminotransferase; BUN, blood urea nitrogen; Cr, creatinine; IL, interleukin; IR, ischemia/reperfusion; LDH, lactate dehydrogenase; MDA, malondialdehyde; ROS, reactive oxygen species; SOD, superoxide dismutase.
\end{abstract}

\section{Introduction}

Liver ischemia/reperfusion (IR) is a complication of liver surgery, especially during liver transplantation which results in inflammation and organ dysfunction (Fondevila

Correspondence to: Mehri Kadkhodaee, Department of Physiology, School of Medicine, Tehran University of Medical Sciences, Tehran 14155-6447, Iran

E-mail:kadkhodm@tums.ac.ir et al. 2003). In addition, recent studies indicate that IRinduced acute liver failure (ALF) causes injuries of distant organs such as heart and lungs by systematic inflammatory responses (Weinbroum et al. 1997). However, the effects of acute liver injury on the induction of kidney injury have so far not been adequately addressed. In clinical settings, if acute kidney injury (AKI) concomitantly occurs in patients with ALF, mortality will significantly increase (Davis et al. 2002; Betrosian et al. 2007). In experimental studies, Behrends et al. in 2007 showed that 
severe hepatic ischemia results in a moderate impairment of renal function in rats but does not trigger an inflammatory response in the kidney and does not result in morphological damage of the kidney (Behrends et al. 2008). However, Lee et al. (2009) showed that AKI rapidly and reproducibly develops in mice after hepatic IR and is characterized by renal tubular necrosis, inflammatory changes and interstitial capillary endothelial apoptosis. Since multiple organ failure continues to be a major cause of death in the surgical intensive care unit, there is a need to explore the extent of renal damage as a result of the liver injury. Researchers believe that remote organ injuries are largely a consequence of the endogenously produced mediators rather than the exogenous factors and toxins (Weinbroum et al. 1999). Thus, studies of the injury to individual organs during distant organ injuries are highly desired. Therefore, in the present study, the effects of induction of 90 min partial hepatic ischemia on the renal functional, histological, oxidative stress and inflammatory indices were studied during a short and a longer period of reperfusion.

\section{Materials and Methods}

\section{Animals}

Male Albino rats (250-300 g) were housed in standard conditions ( $12 \mathrm{~h} \mathrm{light/day} \mathrm{cycle} \mathrm{with} 20-22^{\circ} \mathrm{C}$ temperature and $40-50 \%$ humidity) and had free access to commercial chow and water. All procedures described here had prior approval from the University Animal Ethics Committee and are in accordance with the Helsinki Declaration, as revised in Edinburgh 2000.

\section{Surgical protocol}

Four groups of rats $(n=8)$ were included in this study and randomly assigned into one of the experimental groups: 1) $90 \mathrm{~min}$ liver ischemia and $4 \mathrm{~h}$ reperfusion (IR $4 \mathrm{~h}$ ); 2 ) Sham-operated rat for group one; 3) 90 min liver ischemia and $24 \mathrm{~h}$ reperfusion (IR 24h); and 4) Sham-operated rat for group three.

Rats were placed on a warming pad and were anaesthetized by intraperitoneal injection of sodium pentobarbital $(75 \mathrm{mg} / \mathrm{kg}$ followed by $6 \mathrm{mg} / \mathrm{kg}$ per h intravenously, Sigma-Aldrich, Steinheim, Germany). A tracheotomy was performed to facilitate free breathing. After tracheotomy, the tail vein was cannulated (using Venflon 22GA, 0.98IN, ID $0.8 \mathrm{~mm}$, Helsingborg, Sweden) and $0.9 \%$ normal saline was infused to maintain euvolemia. The right femoral artery was cannulated (Vasculon 25GA, 0.75IN, ID $0.6 \mathrm{~mm}$ ) and connected to a pressure transducer (MLT 0380, ADInstru- ments, Castle Hill, Australia) for mean arterial pressure and heart rate monitoring, which were recorded using a PowerLab/4sp data acquisition system (Software Chart, version 5.0.1, ADInstruments).

Body temperature was maintained at $37 \pm 1^{\circ} \mathrm{C}$. A midline laparatomy was performed and the liver artery and portal vein was carefully separated from the around tissue. To induce partial ischemia, portal vein and liver artery perfusing left and median lobes of the liver were occluded by a non-traumatic micro-vascular clip (Biemerclip, Aesculap, Germany). Sham operated animals underwent identical surgical treatment, including isolation of liver artery. However, artery occlusion was not performed. After completion of the surgery, rats were allowed to stabilize for $30 \mathrm{~min}$.

Ninety minutes of liver ischemia was induced and rats were euthanized 4 or $24 \mathrm{~h}$ after surgery. Blood samples were collected and centrifuged at $4000 \times g$ for $10 \mathrm{~min}$ at $4^{\circ} \mathrm{C}$, and serum was collected for chemical analysis. Kidney tissues were fixed in formalin ( $10 \%$ phosphate-buffered, $\mathrm{pH}=7.4$ ) for histological evaluations. Remainders of renal tissues were partly washed in cold phosphate-buffered saline and partly snap-frozen in liquid nitrogen. The samples were stored at $-70^{\circ} \mathrm{C}$ until further study.

\section{Biochemical assay}

Blood urea nitrogen (BUN) and creatinine (Cr) were used as renal functional indices. Blood concentrations of $\mathrm{LDH}$ (lactate dehydrogenase), AST (aspartate amino-transferase), ALT (alanine amino-transferase) and ALK (alkaline phosphatase), were determined by commercially available kits to confirm liver IR-induced injury.

\section{Measurement of renal oxidative stress markers}

The tissue MDA (malondialdehyde) level was determined by method of Esterbauer and Cheeseman (Esterbauer and Cheeseman 1990) based on its reaction with thiobarbituric acid at $90-100^{\circ} \mathrm{C}$ and measurement of the absorbance at $532 \mathrm{~nm}$. MDA reacts with thiobarbituric acid (TBA) and produces a pink pigment which has a maximum absorption at $532 \mathrm{~nm}$. The value of each sample was obtained from the standard curve and was expressed as $\mathrm{nmol} / \mathrm{g}$ tissue. SOD (superoxide dismutase) activity was measured according to Paoletti et al. method (Paoletti and Mocali 1990). In this assay, superoxide anion is generated from molecular oxygen in the presence of EDTA, manganese (II) chloride, and mercaptoethanol. Nicotinamide adenine dinucleotide phosphate (NADPH) oxidation is linked to the availability of superoxide anions in the medium. Catalase activity was measured in renal homogenate fractions by spectrophotometric analysis (at $240 \mathrm{~nm}$ ) of the 
rate of hydrogen peroxide decomposition according to the method of Aebi (1984).

\section{Determination of cytokine concentrations}

Cytokines concentrations, including IL-6 and IL-10, were measured in serum using enzyme-linked immunosorbent assay (ELISA) method (rat IL-10 and IL-6; ELISA kit, Diaclone A tepnel Company, Besankon, France).

\section{Histological analysis}

After formalin fixation (10\% phosphate-buffered) and dehydration, paraffin-embedded renal sections $(4 \mu \mathrm{m})$ were stained by hematoxylin and eosin. Tubules were evaluated for the presence of degenerative changes (vacuolization), tubular dilatation, luminal debris and cast formation, and loss of brush borders from proximal tubules.

A

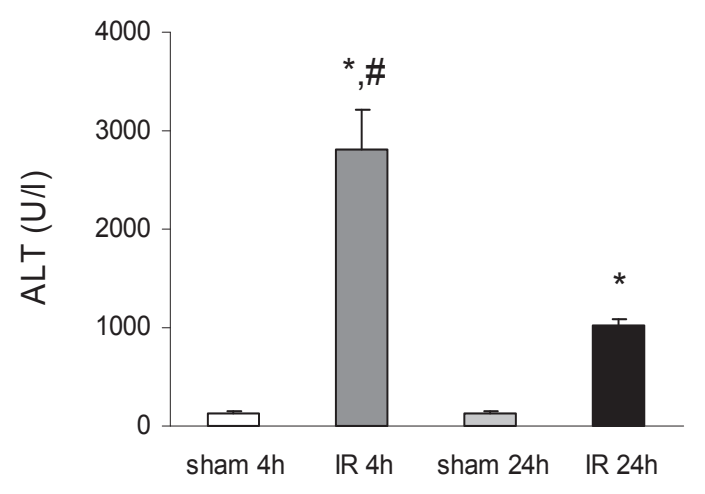

C

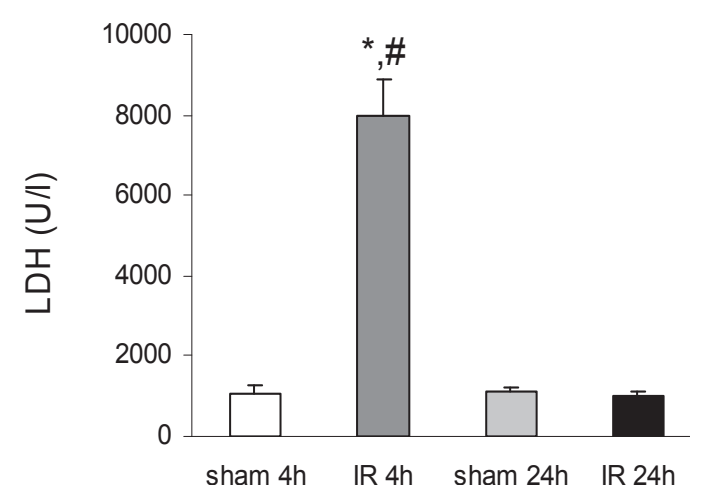

Statistical analysis

The results are given as mean \pm SEM. Statistical analysis was performed by analysis of variance using a post-hoc Tukey test. The null hypothesis was rejected at the 0.05 level of significance. SPSS 11.0 software (Chicago, IL, USA) was used for data analysis.

\section{Results}

\section{Liver functional changes}

Liver ischemia for $90 \mathrm{~min}$ followed by $4 \mathrm{~h}$ reperfusion resulted in significant changes in liver functional indices, as was demonstrated by significant increase in serum concentrations of ALT, AST and LDH compared to sham-operated rats (Fig. 1). By $24 \mathrm{~h}$ of reperfusion the concentrations were decreased (group

B

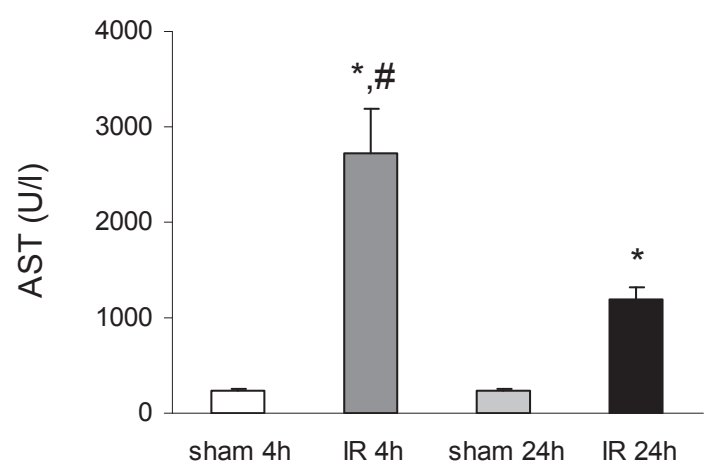

$\mathrm{D}$

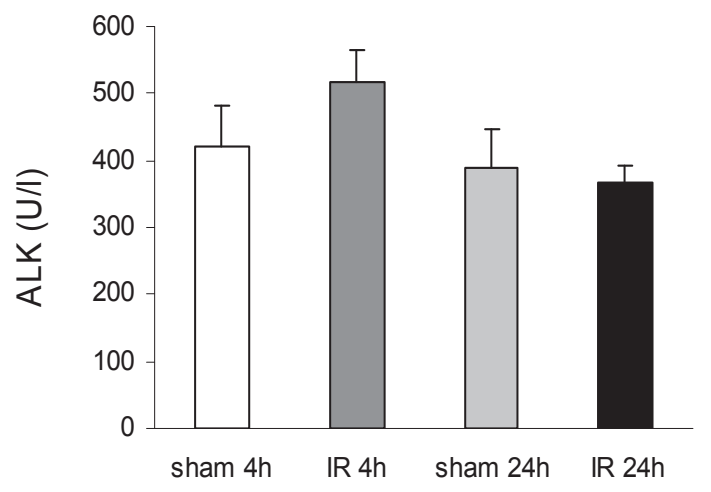

Figure 1. Changes in serum ALT (A), AST (B), LDH (C) and ALK (D) following 4 or $24 \mathrm{~h}$ of reperfusion in liver IR (ischemia-reperfusion) group, comparing to the sham group. Results are the mean \pm S.E.M. ${ }^{*} p<0.05$ compared with sham group, \# $p<0.05$ compared with IR 24h group; $(n=8)$. 
IR 24h) significantly compared to $4 \mathrm{~h}$ reperfusion (IR $4 \mathrm{~h}$ ) group (Fig. 1A, B and C). ALK activity was higher in IR 4 h group comparing to other groups, but this was not significant (Fig. 1D).

\section{Renal functional changes}

Liver ischemia for $90 \mathrm{~min}$ followed by $4 \mathrm{~h}$ reperfusion resulted in a significant rise in concentration of BUN compared to sham-operated rats (Fig. 2). By 24 h of reperfusion (group IR 24h) the concentrations were decreased significantly compared to IR 4 h group (Fig. 2A). There were no significant changes in serum creatinine between groups (Fig. 2B).

\section{Renal oxidative status changes}

Liver ischemia for $90 \mathrm{~min}$ followed by $4 \mathrm{~h}$ reperfusion resulted in significant changes in renal oxidative stress markers including tissue MDA levels (Fig. 3A), SOD (Fig. 3B) and catalase activities (Fig. 3C) compared to sham-operated rats. By $24 \mathrm{~h}$ of reperfusion MDA level was decreased significantly but was still significantly different from sham-operated levels. The SOD and catalase activities significantly increased by $24 \mathrm{~h}$ reperfusion and reached to the sham-operated rats.

\section{Serum inflammatory cytokine changes}

Liver ischemia for 90 min followed by $4 \mathrm{~h}$ reperfusion resulted in significant changes in serum IL-6 (Fig. 4A) and IL-10 (Fig. $4 \mathrm{~B})$ compared to sham-operated rats. By $24 \mathrm{~h}$ of reperfusion (group IR 24h) the concentrations were decreased significantly compared to IR 4 h group (Figs. $4 \mathrm{~A}$ and B).

\section{Histological evaluations}

As it is shown in Fig. 5, there is no detectable change by light microscopy in the kidney of sham group (Fig. 5A). Cells are healthy, nuclei are normal, the brush borders are intact and tubular lumens are open. In group IR 4h (Fig. 5B), there is significant alteration in the kidney histology, including formation of luminal debris, flattening of tubular cells, cellular vacuolization, irregularities in cytoplasm and nuclei of epithelial cells and loss of brush borders. In IR $24 \mathrm{~h}$ group (Fig. 5C), there is less structural damage comparing to IR 4 h group. This was demonstrated by less cytoplasmic vacuolation, lower tubular debris in a way that most of the tubules are open. As a conclusion of histological evaluations, although ischemia and $4 \mathrm{~h}$ reperfusion resulted in significant morphological damage, but in a longer reperfusion time, improvement in histology is seen.

\section{Discussion}

Reperfusion injury induces a systematic inflammatory response which results in the burst formation of ROS (reactive oxygen species) (Kadkhodaee et al. 1996). This augments local tissue damage or may affect organs remote from the site of IR (Serteser et al. 2002). In recent years there have been many efforts on revealing the mechanisms involved in remote organ injury. There is a crosstalk between the kidney and the liver (Li et al. 2009) and the severity of liver injury and hepatocyte death has been associated with the severity of kidney injury. In 2009, we showed that renal IR induces changes in liver histology, function and inflammatory status, which led to a reduction in hepatic antioxidant capacity (Kadkhodaee et al. 2009). This was confirmed by another study demonstrating that either acute kidney ischemia or renal failure as a result of total nephrectomy activates oxidative stress and promotes inflammation, apoptosis, and tissue damage in hepatocytes (Golab et al. 2009).

Hepatic IR-induced liver injury is a very common clinical phenomenon and usually leads to remote damage to other organs including heart and lungs (Wanner et al. 1996; Tanaka
A

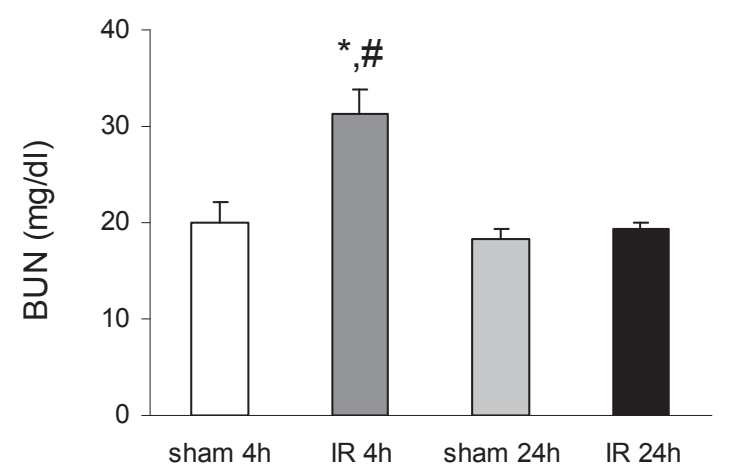

B

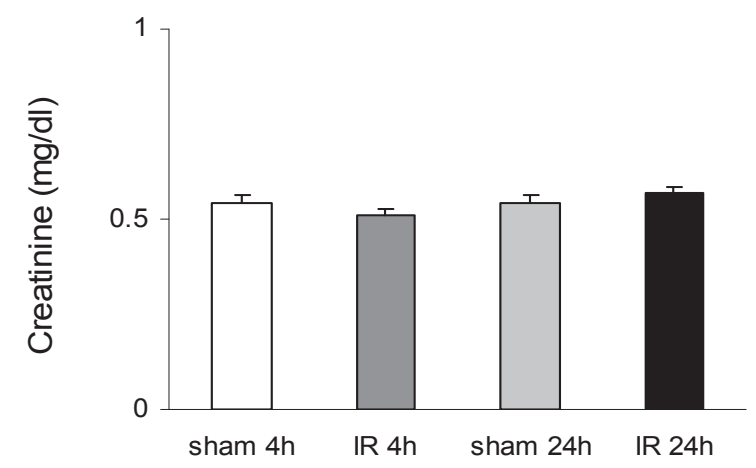

Figure 2. Changes in blood urea nitrogen (BUN, A) and serum ceatinine (B) following 4 or $24 \mathrm{~h}$ of reperfusion in liver IR (ischemiareperfusion) group, comparing to the sham group. Results are the mean \pm S.E.M. ${ }^{*} p<0.05$ compared with sham group; $(n=8)$. 
A

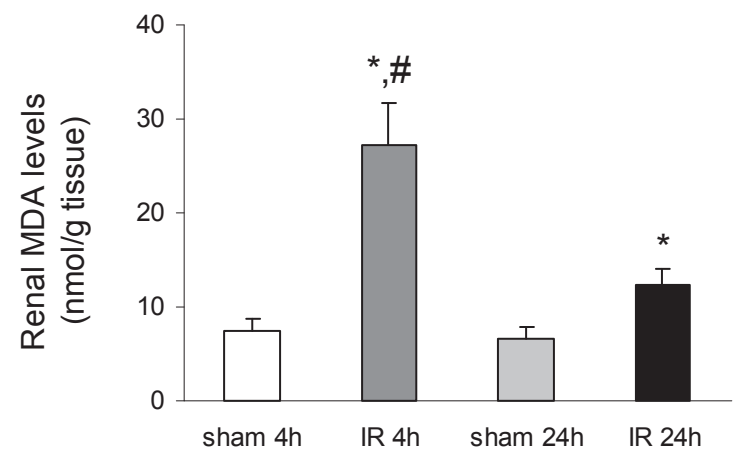

$\mathrm{C}$

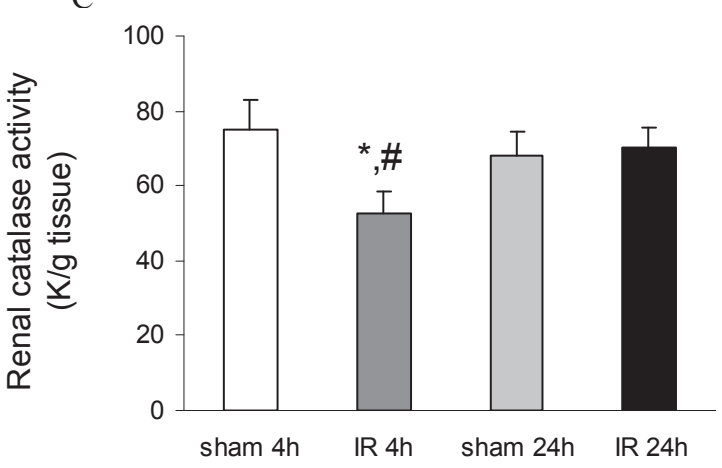

et al. 2007). Renal injury associated with liver disease is also an extensively encountered clinical problem of varied etiology and high mortality (Sural et al. 2000). However, the pathogenesis of AKI associated with liver disease is poorly understood, largely because of the low number of relevant experimental studies. Even in these few available data, discrepant results are evident which may be related to the different models used. These include different animal species,
B

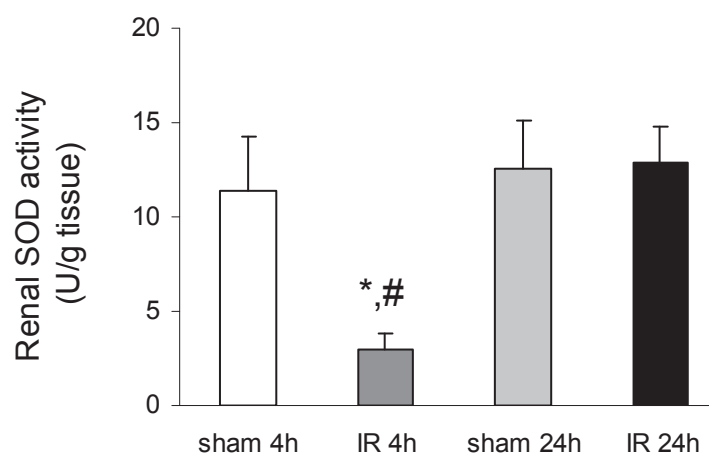

Figure 3. Changes in renal tissue MDA levels (A), SOD (B) and catalase activities (C) following 4 or $24 \mathrm{~h}$ of reperfusion in liver IR (ischemia-reperfusion) group, comparing to the sham group. Results are the mean \pm S.E.M. ${ }^{\star} p<0.05$ compared with sham group, $\# p<0.05$ compared with IR $24 \mathrm{~h}$ group; $(n=8)$.

partial or total liver ischemia, 45 to $90 \mathrm{~min}$ ischemia and different reperfusion periods which were used or applied.

There are several reports indicating that hepatic IR alone does not cause significant renal damage. Behrends et al. (2008) demonstrated that acute hepatic injury results in only transiently impaired renal function but does not trigger an inflammatory response of the kidney itself and does not cause subsequent kidney morphological damage. The study

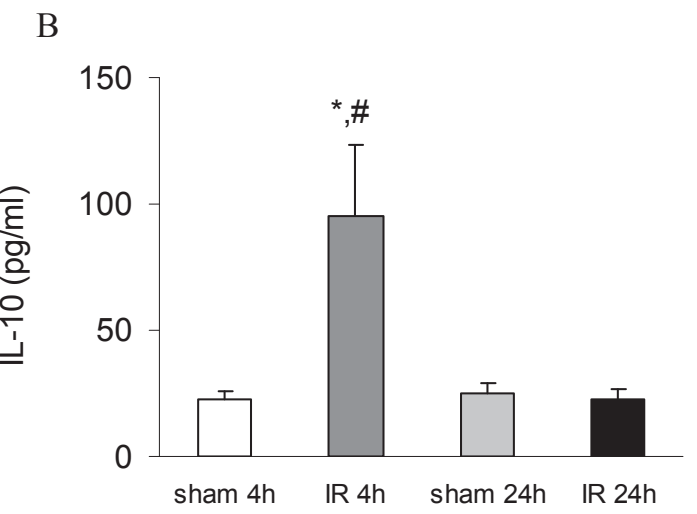

Figure 4. Serum IL-6 (A) and IL-10 (B) in experimental groups following 4 or $24 \mathrm{~h}$ of reperfusion in liver IR (ischemia-reperfusion) group, comparing to the sham group. Results are the mean \pm S.E.M. ${ }^{*} p<0.05$ compared with sham group, \# $p<0.05$ compared with IR 24h group; $(n=8)$. 
A

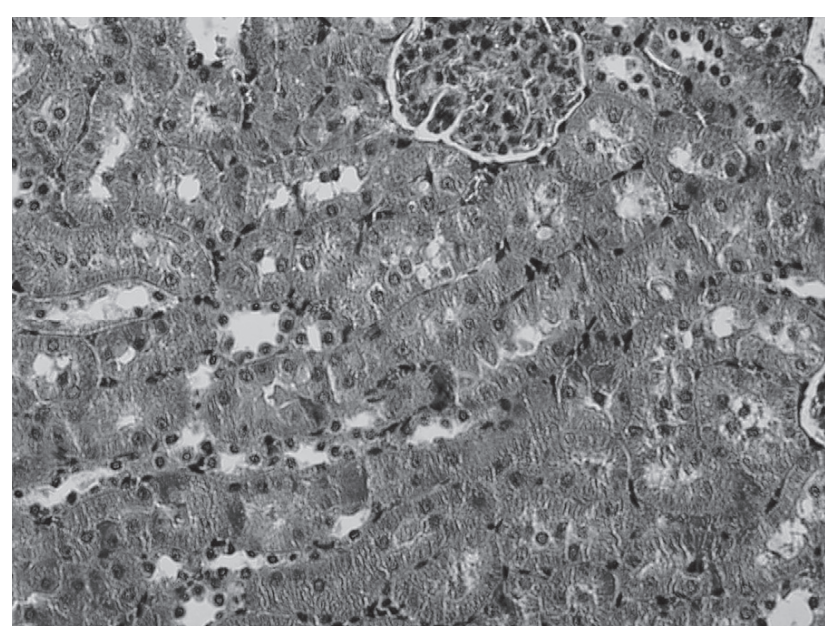

C

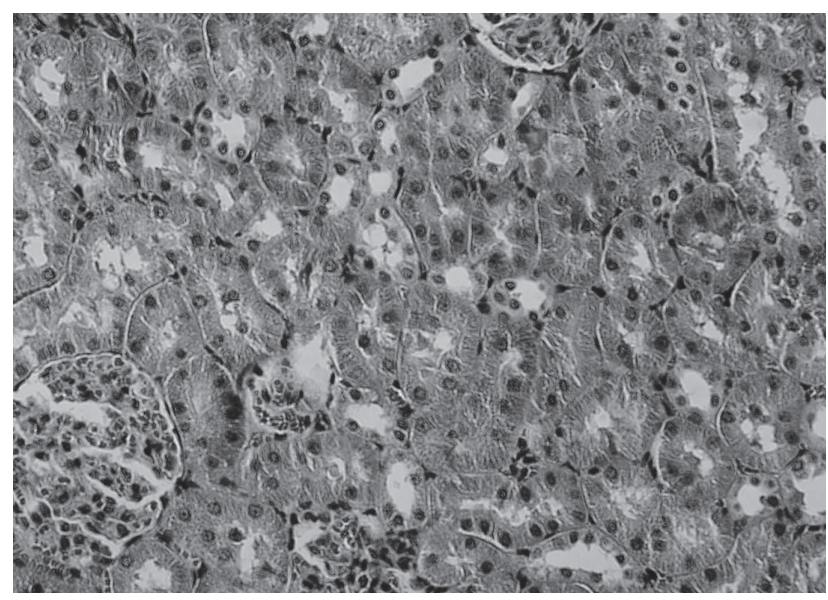

B

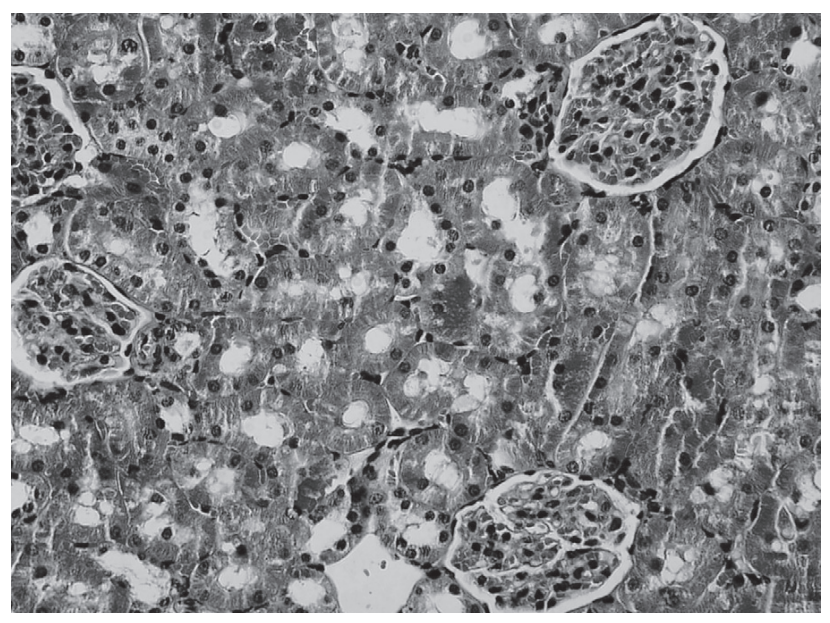

Figure 5. Histological evaluation of the renal tissues in different groups. In sham group (A), there is no detectable change by light microscopy. In IR $4 \mathrm{~h}$ reperfusion group (B), there is significant alterations in the kidney histology, including formation of luminal debris, cellular vacuolization and loss of brush borders. In IR $24 \mathrm{~h}$ group $(\mathbf{C})$, there is less structural damage comparing to IR $4 \mathrm{~h}$ group. Magnification $\times 400$; Hematoxylin and Eosin staining.

of Polat et al. (2006) on rats showed no or little morphological damage of the kidney subjected to $45 \mathrm{~min}$ total hepatic ischemia followed by 1 hour of reperfusion. On the other hand, Lee et al. (2009) showed that mice subjected to 60 min partial liver ischemia develop severe AKI in less than $24 \mathrm{~h}$. During reperfusion following $60 \mathrm{~min}$ of liver ischemia, numerous changes were found including biochemical and renal morphological injury as well as increase in endotoxin, lipid peroxide, and lysosomal enzymes in the blood (Kudo et al. 1993).

Remote organ injuries are largely consequences of the endogenously produced mediators rather than the exogenous factors and toxins (Weinbroum et al. 1999). Inhibition of inducible nitric oxide synthase has shown to be protective in amelioration of lung injury after gut ischemia-reperfusion (Uchida et al. 2007). Inflammatory changes in the kidney after hepatic IR may include neutrophil infiltration of the interstitium and upregulation of several proinflammatory mRNAs such as tumor necrosis factor-alpha, keratinocytederived cytokine, monocyte chemotactic protein-1, macrophage inflammatory protein-2 and intercellular adhesion molecule-1 (Lee et al. 2009). Park et al. (2009) showed that over expression of human heat shock protein 27 protects against hepatic injury and AKI associated with liver IR in vivo.

Intra-renal vasoconstriction as a result of circulatory damaging mediators is discussed as the initiating event to induce secondary renal dysfunction in the setting of hepatic failure (hepatorenal syndrome, HRS). HRS is an important mechanism of end-organ dysfunction in kidney-liver crosstalk, where dysregulation of vasoactive mediators leads to acute kidney injury. However, the severity of the kidney damage depends on the amount of liver injury and there is always the possibility that high 
intensity of the liver injury induce the irreversible changes in the kidneys.

In the present study, partial ischemia of liver for $90 \mathrm{~min}$ followed by $4 \mathrm{~h}$ reperfusion caused a significant reduction in hepatic function demonstrated by plasma increase in liver enzyme activities. However, the liver injury reduced by the increase in reperfusion time (Yabe et al. 2001; Tanaka et al. 2006), but had still significant differences in comparison with control group which indicates severe and persistent liver tissue injury as a result of direct IR of this organ (Zhang et al. 2007). Renal function was also reduced, demonstrated by an increase in BUN and accompanied by renal oxidative stress which was demonstrated by an increase in renal MDA level and a decrease in antioxidants concentrations (SOD and catalase activities) and an increase in inflammatory cytokines. Histological changes showed renal tubular injuries which may be the effects of oxidative or inflammatory factors released/stimulated during liver ischemia. These findings are in agreement with the Mutlu et al. (2002) study in which intestinal ischemia-reperfusion led to renal dysfunction and ROS were introduced as the main causative factors of injury. They demonstrated the reversibility of the renal changes after $24 \mathrm{~h}$ in which MDA and BUN had returned to normal levels. In the present study, $24 \mathrm{~h}$ after ischemia there were alleviation of renal functional and histological damages and reduction in oxidative and inflammatory changes comparing to $4 \mathrm{~h}$ reperfusion group. These improvements suggest that if there are moderated levels of remote changes to the kidney, physiological systems may modulate the injuries by time.

Another important point in the crosstalk between kidneys and liver is that kidneys seem to be more tolerant to remote injury comparing to liver, since lower periods of renal ischemia (30-60 min) are known to significantly affect the liver, but longer periods (60-75 $\mathrm{min}$ ) of liver ischemia are not. This may be due to the higher degree of sensitivity of liver to remote oxidative stress (Behrends et al. 2008) or the fact that kidney acts as a more potent trigger of remote injury. Histological changes we observed in liver of rats subjected to $45 \mathrm{~min}$ of renal IR were more severe (Golab et al. 2009) than the effects of $90 \mathrm{~min}$ of liver IR on kidney structure as it was demonstrated in the present study.

In recent years, remote ischemic conditionings are introduced as noninvasive and applicable methods against IR injuries. In a recent study, we demonstrated the protective effects of remote per- and post-conditioning on IR-induced renal damage (Kadkhodaee et al. 2011). Nitric oxide may also be an essential mediator of the protection afforded by remote ischemic preconditioning against IR injury. The mechanisms underlying this protection involve preservation of the tissue structure and maintenance of blood flow through the organ microcirculation (Abu-Amara et al. 2011).
It should also be noted that, in the experimental studies on remote organ injury, the extra-organ is normal at the onset of local organ IR injury. However, this is almost clinically irrelevant, since in most situations in patients we encounter to the dysfunction or failure of two or more organs namely multi-organ failure. This must be considered when the results of experimental studies are interpreted for use in clinical settings.

\section{Conclusion}

This study showed that liver IR damage cause renal injury including functional, inflammatory and oxidative status changes. The remote kidney damage was then improved by continuing reperfusion from 4 to $24 \mathrm{~h}$. However, the phenomenon of remote organ injury has to be kept in mind when performing a liver surgery and care should be taken to protect other organs remote from the site of IR. Future studies are needed to build on these observations in order to develop strategies to improve outcomes in the patients with multi-organ dysfunctions.

Acknowledgements. This study was supported by a grant from Tehran Medical Sciences University (to MK).

\section{References}

Abu-Amara M., Yang S. Y., Quaglia A., Rowley P., de Mel A., Tapuria N., Seifalian A., Davidson B., Fuller B. (2011): Nitric oxide is an essential mediator of the protective effects of remote ischaemic preconditioning in a mouse model of liver ischaemia/reperfusion injury. Clin. Sci. (Lond) 121, 257-266 http://dx.doi.org/10.1042/CS20100598

Aebi H. (1984): Catalase in vitro. Methods. Enzymol. 105, $121-126$ http://dx.doi.org/10.1016/S0076-6879(84)05016-3

Behrends M., Hirose R., Park Y. H., Tan V., Dang K., Xu F., Park S. H., Niemann C. U. (2008): Remote renal injury following partial hepatic ischemia/reperfusion injury in rats. J. Gastrointest. Surg. 12, 490-495

http://dx.doi.org/10.1007/s11605-007-0252-6

Betrosian A. P., Agarwal B., Douzinas E. E. (2007): Acute renal dysfunction in liver diseases. World. J. Gastroenterol. 13, 5552-5559

Davis C. L., Gonwa T. A., Wilkinson A. H. (2002): Pathophysiology of renal disease associated with liver disorders: implications for liver transplantation. Part I. Liver Transpl. 8, 91-109 http://dx.doi.org/10.1053/jlts.2002.31516

Esterbauer H., Cheeseman K. H. (1990): Determination of aldehydic lipid peroxidation products: malonaldehyde and 4-hydroxynonenal. Methods. Enzymol. 186, 407-421 http://dx.doi.org/10.1016/0076-6879(90)86134-H

Fondevila C., Busuttil R. W., Kupiec-Weglinski J. W. (2003): Hepatic ischemia/reperfusion injury--a fresh look. Exp. Mol. Pathol. 74, 86-93 http://dx.doi.org/10.1016/S0014-4800(03)00008-X 
Golab F., Kadkhodaee M., Zahmatkesh M., Hedayati M., Arab H., Schuster R., Zahedi K., Lentsch A. B., Soleimani M. (2009): Ischemic and non-ischemic acute kidney injury cause hepatic damage. Kidney Int. 75, 783-792 http://dx.doi.org/10.1038/ki.2008.683

Kadkhodaee M., Golab F., Zahmatkesh M., Ghaznavi R., Hedayati M., Arab H. A., Ostad, S. N., Soleimani M. (2009): Effects of different periods of renal ischemia on liver as a remote organ. World. J. Gastroenterol. 15, 1113-1118 http://dx.doi.org/10.3748/wjg.15.1113

Kadkhodaee M., Hanson G. R., Towner R. A., Endre Z. H. (1996): Detection of hydroxyl and carbon-centred radicals by EPR spectroscopy after ischaemia and reperfusion of the rat kidney. Free. Radic. Res. 25, 31-42 http://dx.doi.org/10.3109/10715769609145654

Kadkhodaee M., Seifi B., Najafi A., Sedaghat Z. (2011): First report of the protective effects of remote per- and postconditioning on ischemia/reperfusion-induced renal injury. Transplantation 92, e55 http://dx.doi.org/10.1097/TP.0b013e31823411f8

Kudo Y., Egashira T., Takayama F., Yamanaka Y., Shimada T. (1993): Investigation of the renal injury caused by liver ischemia-reperfusion in rats. Arch. Toxicol. 67, 502-509 http://dx.doi.org/10.1007/BF01969922

Lee H. T., Park S. W., Kim M., D’Agati V. D. (2009): Acute kidney injury after hepatic ischemia and reperfusion injury in mice. Lab. Invest. 89, 196-208 http://dx.doi.org/10.1038/labinvest.2008.124

Li X., Hassoun H. T., Santora R., Rabb H. (2009): Organ crosstalk: the role of the kidney. Curr. Opin. Crit. Care. 15, 481-487 http://dx.doi.org/10.1097/MCC.0b013e328332f69e

Mutlu G., Abbasoglu L., Dogru-Abbasoglu S., Solakoglu S., Bulut M. (2002): Morphologic changes and lipid peroxidation in renal tissues of young rats following intestinal ischemia-reperfusion. Pediatr. Surg. Int. 18, 337-340 http://dx.doi.org/10.1007/s00383-002-0834-z

Paoletti F., Mocali A. (1990): Determination of superoxide dismutase activity by purely chemical system based on NAD $(\mathrm{P}) \mathrm{H}$ oxidation. Methods Enzymol. 186, 209-220 http://dx.doi.org/10.1016/0076-6879(90)86110-H

Park S. W., Chen S. W., Kim M., D‘Agati V. D., Lee H. T. (2009): Human heat shock protein 27 -overexpressing mice are protected against acute kidney injury after hepatic ischemia and reperfusion. Am. J. Physiol. Renal. Physiol. 297, F885-894 http://dx.doi.org/10.1152/ajprenal.00317.2009

Polat C., Tokyol C., Kahraman A., Sabuncuoglu B., Yilmaz S. (2006): The effects of desferrioxamine and quercetin on hepatic ischemia-reperfusion induced renal disturbance. Prostaglandins Leukot. Essent. Fatty Acids 74, 379-383 http://dx.doi.org/10.1016/j.plefa.2006.03.007
Serteser M., Koken T., Kahraman A., Yilmaz K., Akbulut G., Dilek O. N. (2002): Changes in hepatic TNF-alpha levels, antioxidant status, and oxidation products after renal ischemia/reperfusion injury in mice. J. Surg. Res. 107, 234-240 http://dx.doi.org/10.1006/jsre.2002.6513

Sural S., Sharma R. K., Gupta A., Sharma A. P., Gulati S. (2000): Acute renal failure associated with liver disease in India: etiology and outcome. Ren. Fail. 22, 623-634 http://dx.doi.org/10.1081/JDI-100100903

Tanaka Y., Chen C., Maher J. M., Klaassen C. D. (2006): Kupffer cellmediated downregulation of hepatic transporter expression in rat hepatic ischemia-reperfusion. Transplantation 82, 258-266 http://dx.doi.org/10.1097/01.tp.0000226243.69023.54

Tanaka Y., Maher J. M., Chen C., Klaassen C. D. (2007): Hepatic ischemia-reperfusion induces renal heme oxygenase-1 via NF-E2-related factor 2 in rats and mice. Mol. Pharmacol. 71, $817-825$ http://dx.doi.org/10.1124/mol.106.029033

Uchida K., Mishima S., Ohta S., Yukioka T. (2007): Inhibition of inducible nitric oxide synthase ameliorates lung injury in rats after gut ischemia-reperfusion. J. Trauma 63, 603-607 http://dx.doi.org/10.1097/TA.0b013e3181271b0b

Wanner G. A., Ertel W., Muller P., Hofer Y., Leiderer R., Menger M. D., Messmer K. (1996): Liver ischemia and reperfusion induces a systemic inflammatory response through Kupffer cell activation. Shock 5, 34-40 http://dx.doi.org/10.1097/00024382-199601000-00008

Weinbroum A. A., Hochhauser E., Rudick V., Kluger Y., Karchevsky E., Graf E., Vidne B. A. (1999): Multiple organ dysfunction after remote circulatory arrest: common pathway of radical oxygen species? J. Trauma 47, 691-698 http://dx.doi.org/10.1097/00005373-199910000-00013

Weinbroum A. A., Hochhauser E., Rudick V., Kluger Y., Sorkine P., Karchevsky E., Graf E., Boher P., Flaishon R., Fjodorov D., Niv D., Vidne, B. A. (1997): Direct induction of acute lung and myocardial dysfunction by liver ischemia and reperfusion. J. Trauma 43, 627-633 http://dx.doi.org/10.1097/00005373-199710000-00011

Yabe Y., Kobayashi N., Nishihashi T., Takahashi R., Nishikawa M., Takakura Y., Hashida M. (2001): Prevention of neutrophilmediated hepatic ischemia/reperfusion injury by superoxide dismutase and catalase derivatives. J. Pharmacol. Exp. Ther. 298, 894-899

Zhang W., Wang M., Xie H. Y., Zhou L., Meng X. Q., Shi J., Zheng S. (2007): Role of reactive oxygen species in mediating hepatic ischemia-reperfusion injury and its therapeutic applications in liver transplantation. Transplant. Proc. 39, 1332-1337 http://dx.doi.org/10.1016/j.transproceed.2006.11.021

Received: October 10, 2011

Final version accepted: December 21, 2011 